\title{
The management of Eisenmenger syndrome in the modern treatment era: a case report
}

\section{To the Editor:}

A 41-yr-old female with a history of Eisenmenger syndrome (ES) was referred to the Royal Brompton Hospital, London, UK in 2002 for tertiary care.

The patient had undergone closure of a large ventricular septal defect (VSD) via medial sternotomy at the age of 8 yrs elsewhere. At the time, the patient had a left-to-right shunt with high pulmonary artery saturation of $87 \%$ and low aortic saturation of $93 \%$ in air and evidence of pulmonary vascular disease, with systemic pulmonary artery pressure and a calculated pulmonary vascular resistance (PVR) of 6 Wood units. Her post-operative course was somewhat complicated and the VSD patch dehisced. The patient remained relatively well until her teenage years, when her condition deteriorated and she was diagnosed with ES. At 22 yrs she was sterilised following termination of an unplanned pregnancy; her risk of maternal mortality was quoted as $30-50 \%$ at that time. She was put on a waiting list for transplantation during the 1980s, but was relisted to a "wait/see" status in the 1990s as she seemed to be doing well. Her medical history included recurrent transient ischaemic attacks and iron deficiency anaemia, and the presence of paroxysmal atrial flutter.

Following assessment at the Royal Brompton Hospital, the patient was included in a pilot study of bosentan in congenital heart disease. Treatment was initiated with $62.5 \mathrm{mg}$ bosentan twice daily, and her dose was uneventfully uptitrated to $125 \mathrm{mg}$ twice daily after 4 weeks. There was no drop in resting oxygen saturations during initiation. Transient ankle oedema occurred during the first week of treatment, but this resolved spontaneously by 1 month. After 3 months of treatment, the patient showed marked improvement; her oxygen saturations had improved from $83 \%$ to $87 \%$ in air, blood pressure was stable (99/70 from 114/62 mmHg), her 6-min walk distance (6MWD) had increased from 340 to $495 \mathrm{~m}$, and her World Health Organization functional class (WHO FC) had improved from class III to II. Pulmonary blood flow had increased from 2.52 to 3.37 $\mathrm{L} \cdot \mathrm{min}^{-1}$, and her cardiac index from 1.60 to $2.14 \mathrm{~L} \cdot \mathrm{min}^{-1} \cdot \mathrm{m}^{-2}$. $1 \mathrm{yr}$ after initiation of bosentan, the patient continued to do well, with marked improvements in her quality of life. Prior to bosentan therapy, the patient reported that she could not climb stairs, needed to use a mobility scooter and was afraid to leave the house on her own. Following treatment, she reported that she could climb two flights of stairs, was able to walk comfortably for "about 2 miles" and had returned to part-time work.

After $3.5 \mathrm{yrs}$ on bosentan, the patient reported increasing dyspnoea and fatigue. Her 6MWD had declined to $370 \mathrm{~m}$. Sildenafil $20 \mathrm{mg}$ three times daily was added to her bosentan therapy. The combination was well tolerated and, after 3 months, her oxygen saturations had improved and her blood pressure was stable, her 6MWD had increased to $410 \mathrm{~m}$, and her Cambridge Pulmonary Hypertension Outcome Review (CAMPHOR) quality of life score also improved.

This case study raises a number of important issues in the management of patients with pulmonary artery hypertension $(\mathrm{PAH})$ associated with congenital heart disease (CHD), and also highlights changes in management strategies for these patients over the past decade or so.

The initial decision to close the VSD in this patient, despite the fact that her very high PVR indicated associated PAH, may not have been made today. At the time this patient underwent the procedure, there were no studies on reversibility of $\mathrm{PAH}$ in association with $\mathrm{CHD}$, and long-term outcomes were uncertain. In current practice, the importance of reversibility is understood, and referral for such a closure depends on the status of the pulmonary circulation. For patients with significant VSDs, early repair is crucial as closure in later stage disease, in which pulmonary vascular disease is advanced and PVR is markedly elevated, is associated with a high risk of sustained pulmonary hypertension, right heart failure, and hypertensive crises immediately after surgery [1]. There are limited data on which to base a definition of a "safe" upper limit for PVR when considering surgical repair, although $<6$ Wood units after vasoreactivity testing is generally considered appropriate [2]. Current grown-up congenital heart disease $(\mathrm{GUCH})$ guidelines recommend that patients with VSD and PAH should be considered for surgery when there is still net left-to-right shunt (Qp/Qs >1.5) present and PVR is less than two-thirds of systemic vascular resistance [3]. By current criteria, our patient's PVR of 6 Wood units would place her in a "grey zone" where consensus on optimal management is not so well defined. However, with advances in the medical management of such patients, and in particular the availability of $\mathrm{PAH}$-specific therapies with proven efficacy in patients with PAH-CHD, it is less likely that a patient in this situation would be subjected to the risks associated with surgical repair. The ability of PAH-specific therapies to lower PVR, including patients with near-systemic pulmonary pressures previously thought to have irreversible pulmonary vascular disease [1,4], has led to interest in their use preoperatively in order to improve the suitability of a patient for surgery: the treat-and-repair approach. While current GUCH guidelines state that surgery should be considered in patients with VSD and PAH who have a left-to-right shunt and pulmonary artery pressure or PVR less than two-thirds of systemic values following targeted PAH therapy, they do not discuss the treat-and-repair approach in depth [3]. Although there are a number of short-term reports in the literature regarding the successful use of this strategy [5-9], until more 
data are available, and in particular long-term outcome results, the place of this approach in the management of patients with "inoperable" PAH-CHD remains to be proven. Once a left-toright shunt has reversed and ES has developed, repair is clearly contraindicated [3].

Pregnancy is contraindicated in females with PAH because of the prohibitively high rates of maternal mortality associated with this condition [10]. A recent systematic review found that, although maternal morbidity has decreased significantly over the past decade compared with previous decades, a change associated with the availability of PAH-specific therapies, it still remains extremely high [11]. In the case of patients with $\mathrm{PAH}-$ CHD, the mortality rate in this study was $28 \%$ (from $36 \%$ in previous decades), with the vast majority of deaths occurring within the first month after delivery due to right heart failure [11]. In patients with PAH-CHD, the systemic vasodilation induced by pregnancy together with the increase in cardiac output may enhance right-to-left shunting, exacerbating preexisting hypoxia and leading to further pulmonary vasoconstriction. Additional stress occurring during labour and delivery, when hypercarbia and acidosis may lead to an acute increase in pulmonary hypertension, can also lead to refractory right heart failure, the main cause of death in females with PAH-CHD following pregnancy [11]. Interestingly, the study by BÉDARD et al. [11] found that, unlike in patients with idiopathic PAH, where approximately equal numbers of deaths arose during pregnancy and post-partum, all of the deaths in patients with $\mathrm{PAH}-\mathrm{CHD}$ arose in the post-partum period, possibly reflecting a greater ability of the "trained" (i.e. subjected to chronic pressure overload) right ventricle to cope with pregnancy-induced increases in load. Early advice on contraception and the risks of pregnancy remain vital in females with PAH-CHD. For those females who decide to proceed with pregnancies, a multidisciplinary approach with careful attention to mode of delivery, use of anaesthesia, consideration of thromboprophylaxis and possible use of advanced therapies is required [11].

During the 1980s, at the age of 24 yrs, the patient was put on a waiting list for transplantation, a decision that, again, is unlikely to have been made according to current treatment strategies. Patients with ES have been shown to have a greater prevalence of risk factors and a more difficult post-operative course than other transplant recipients [12]. Although overall survival after transplantation is similar in ES patients as in other transplant recipients, only around half of patients with ES who undergo transplantation are still alive 5 yrs later, and only around one-quarter survive for 10 yrs [12, 13]. Given that the expected survival for patients with ES is higher than this, and considering our patient did relatively well for almost 20 yrs without transplantation, this would have clearly been a very high-risk strategy in her case. Alternative management options are now available, as will be discussed.

Prior to receiving bosentan therapy, the patient suffered from recurrent transient ischaemic attacks and iron deficiency anaemia, both of which were likely to have been linked to the policy at the time for routine venesection in patients with ES. Cyanotic patients are susceptible to compensative secondary erythrocytosis, an adaptive response by the body, triggered by hypoxia to increase the oxygen carrying capacity of the blood through stimulation of bone marrow erythropoiesis, causing a rise in red blood cell mass and haematocrit. However, this can also lead to increased whole blood viscosity, which reduces blood flow and tissue perfusion, leading to symptoms of the "hyperviscosity syndrome" (headache, loss of concentration, muscle weakness and fatigue). The increased risk of cerebrovascular accident (CVA) and myocardial ischaemia associated with hyperviscocity syndrome has led to the use of routine venesection in patients with ES; however, whereas this policy is standard practice in cases of "primary" erythrocytosis such as polycythaemia vera [14], studies have failed to show a similar benefit in patients with cyanotic CHD [15]. In fact, repeated venesections have been shown to result in chronic iron deficiency, which leads to microcytosis and increased whole blood viscosity and so increases the risk of CVA [16]. In addition, the induced iron deficiency decreases the patient's ability to promote secondary erythrocytosis to compensate for cyanosis and so compromises oxygen carrying capacity [17]. Iron deficiency has also been shown to reduce exercise capacity [18]. It should be noted that the symptoms of iron deficiency are similar to those of hypervisocity syndrome. In fact, authors report that the majority of patients with ES and chronic secondary erythrocytosis do not exhibit symptoms of overt hyperviscosity and do not require phlebotomies [19]. A better management strategy may be to ensure that patients do not become iron deficient. In a recent study, 3 months of iron replacement therapy in iron-deficient cyanotic CHD patients was shown to be safe and to result in increases in haemoglobin levels, particularly in those patients with low baseline levels, associated with significant improvement in exercise tolerance and quality of life as measured on the CAMPHOR scale [20]. Furthermore, in a recent study the degree of difference between optimal and actual haemoglobin levels was found to be negatively correlated with exercise capacity, while oxygen saturations only correlated with haemoglobin in patients with optimal levels [21]. The study shows that patients with only a small difference between optimal and actual haemoglobin levels have better exercise capacity, irrespective of oxygen saturation, than those with a greater difference, supporting iron supplementation to ensure haemoglobin levels are as close to optimal as possible in patients with ES.

In line with reported studies in patients with PAH-CHD [4, 21-24], our patient showed good short- and long-term responses to initial bosentan therapy, with improvements in exercise capacity and WHO FC without a negative effect on oxygen saturations.

The improvements seen in our patient's clinical condition were matched by marked improvements in her general quality of life. Treatment with PAH-specific therapies has been shown to improve quality of life in patients with ES $[20,23]$ and such improvements are particularly important in patients with ES. Although mortality is increased in ES relative to the general population, it is relatively low compared with patients with idiopathic PAH [17, 25], largely due to the "safety valve" effect that right-to-left shunting has on the right ventricle; however, this improvement in survival is at the cost of cyanosis which leads to multi-organ involvement.

The patient's positive response to bosentan monotherapy was maintained for over 3 yrs, after which, based on deterioration in her 6MWD and increasing dyspnoea, it was decided to initiate combination therapy by adding sildenafil to her existing regimen. 
This strategy accords with recommendations from recent guidelines for the use of goal-orientated therapy in the treatment of $\mathrm{PAH}$, where additional therapies are added to the regimen of those patients who fail to respond adequately to initial monotherapy [10]. There are an increasing number of controlled and uncontrolled studies reporting the efficacy of combination therapy in PAH [26-32]. It is reasonable to assume that the same approach should be followed for patients with ES, although most studies have predominantly included patients with other forms of $\mathrm{PAH}$ and direct evidence for the efficacy of combination therapy in patients with ES is limited. In a study of $32 \mathrm{PAH}-\mathrm{CHD}$ patients (28 with ES) with clinical worsening despite on-going bosentan therapy, addition of sildenafil was found to be well tolerated, and resulted in significant improvement from baseline after 6 months of therapy in clinical status (WHO FC $2.1 \pm 0.4$ versus $2.9 \pm 0.3 ; \mathrm{p}=0.042)$, $6 \mathrm{MWD}(360 \pm 51$ versus $293 \pm 68 \mathrm{~m}$; $\mathrm{p}=0.005)$, Borg dyspnoea score $(2.9 \pm 1.5$ versus $4.4 \pm 2.3 ; \mathrm{p}=0.036)$ and haemodynamics (pulmonary blood flow $3.4 \pm 1.0$ versus $3.1 \pm 1.2 \mathrm{~L} \cdot \mathrm{min}^{-1} \cdot \mathrm{m}^{-2} ; \mathrm{p}=0.002 ; \mathrm{PVR}$ index $19 \pm 9$ versus $24 \pm 16$ $\mathrm{WU} \cdot \mathrm{m}^{-2} ; \mathrm{p}=0.003$ ) [33]. In a small pilot study including two patients with ES, the addition of tadalafil in patients with deterioration on prostacyclin therapy resulted in improvements in 6MWD and WHO FC [34]. Similar criteria should be evaluated to guide the treat-to-target strategy, although not all of the prognostic parameters suggested for assessing response to treatment in idiopathic PAH are applicable in ES. For example, although WHO FC, 6MWD and brain natriuretic protein (BNP)/ $\mathrm{N}$-terminal pro-BNP plasma levels are likely to be equally useful prognostic indicators to guide treatment in ES, other markers are less useful. For example, right ventricular function is well preserved in ES and the right ventricle is also relieved by the right-to-left shunt; therefore, clinical evidence of right ventricular failure would indicate that the patient's condition is seriously compromised and probably beyond the stage of benefitting from treatment, and syncope is a common finding in ES. Cardiopulmonary exercise testing is a useful prognostic parameter in non-cyanotic PAH-CHD patients but, because of the many complex inter-relationships in the cyanotic patient, its prognostic value in ES is unclear. Right heart catheterisation is not routinely performed in ES patients, so changes in haemodynamic measures such as right atrial pressure and cardiac index are not regularly evaluated, although data suggest that response to nitric oxide [35] and acute reversibility [36] during catheterisation may provide prognostic information.

As well as providing improvements in haemodynamics, exercise capacity, functional status and quality of life, treatment with PAH-specific therapies has been shown to improve survival in patients with various forms of PAH [37], and recent data have shown that this is also the case for patients with ES. In a study of 229 patients with ES treated between 1999 and 2008 at the Royal Brompton Hospital, those patients who received treatment with $\mathrm{PAH}$-specific therapies (prostanoids, endothelin receptor antagonists and phosphodiesterase- 5 inhibitors, singly or in combination) had a significantly lower risk of death over a median follow-up of 4 yrs (unadjusted hazard ratio (HR) $0.21,95 \%$ CI $0.05-0.86$; $p=0.03$ ) [38]. This survival benefit was also seen when baseline differences were adjusted for by the use of propensity scores (HR 0.10, 95\% CI $0.01-0.78 ; p=0.028)$. Of the 52 patients who died during the follow-up period, only two were receiving $\mathrm{PAH}$-specific therapies at time of death; the remaining 66 patients who received $\mathrm{PAH}$-specific therapies (median treatment period $2.4 \mathrm{yrs}$ ) were still alive at the end of follow-up. In contrast, 50 out of 161 patients who did not receive PAH-specific therapies died during follow-up, despite being less impaired at baseline than those who were treated [38].

In conclusion, the management of patients with ES has changed in recent years, with greater understanding of mechanisms associated with the syndrome, and the availability of new medical options. For example, there is no basis for the former practice of routine venesection; in contrast, evidence suggests that iron supplementation, to improve haemoglobin levels, may improve exercise capacity and quality of life. In the current treatment era, effective medical therapies are available that lead to improved haemodynamics, functional status, exercise capacity and quality of life, and convey survival benefits for patients with ES. Given this, there is little reason to risk closure of septal defects in the presence of high pulmonary artery pressure and PVR. Instead, patients should receive advanced PAH therapy indefinitely.

\section{M.A. Gatzoulis}

Adult Congenital Heart Centre and Centre for Pulmonary Hypertension, Royal Brompton Hospital, London, UK.

Correspondence: M.A. Gatzoulis, Royal Brompton Hospital, Sydney Street, London, SW3 6NP, UK. E-mail: m.gatzoulis@ rbht.nhs.uk

Statement of Interest: M.A. Gatzoulis has served on the advisory boards of Actelion UK, Pfizer and GSK, and has received unrestricted educational funds from Actelion UK and Pfizer.

Provenance: Publication of this peer-reviewed article was supported by Actelion Pharmaceuticals Ltd, Switzerland (principal sponsor, European Respiratory Review issue 122).

Acknowledgements: I received editorial assistance from L. Thomas (Elements Communications Ltd, Westerham, UK), supported by Actelion Pharmaceuticals Ltd (Allschwil, Switzerland).

\section{REFERENCES}

1 Diller GP, Gatzoulis MA. Pulmonary vascular disease in adults with congenital heart disease. Circulation 2007; 115: 1039-1050.

2 Gatzoulis MA, Alonso-Gonzalez R, Beghetti M. Pulmonary arterial hypertension in paediatric and adult patients with congenital heart disease. Eur Respir Rev 2009; 18: 154-161.

3 Baumgartner H, Bonhoeffer P, De Groot NM, et al. ESC Guidelines for the management of grown-up congenital heart disease (new version 2010). Eur Heart J 2010; 31: 2915-2957.

4 Galie N, Beghetti M, Gatzoulis MA, et al. Bosentan therapy in patients with Eisenmenger syndrome: a multicenter, double-blind, randomized, placebo-controlled study. Circulation 2006; 114: 48-54.

5 Hoetzenecker K, Ankersmit HJ, Bonderman D, et al. Atrial septal defect repair after a 10-month treatment with bosentan in a patient with severe pulmonary arterial hypertension: a case report. J Thorac Cardiovasc Surg 2009; 137: 760-761. 
6 Kim YH, Yu JJ, Yun TJ, et al. Repair of atrial septal defect with Eisenmenger syndrome after long-term sildenafil therapy. Ann Thorac Surg 2010; 89: 1629-1630.

7 Frost AE, Quiñones MA, Zoghbi WA, et al. Reversal of pulmonary hypertension and subsequent repair of atrial septal defect after treatment with continuous intravenous epoprostenol. J Heart Lung Transplant 2005; 24: 501-503.

8 Hirabayashi A, Miyaji K, Akagi T. Continuous epoprostenol therapy and septal defect closure in a patient with severe pulmonary hypertension. Catheter Cardiovasc Interv 2009; 73: 688-691.

9 Schwerzmann M, Zafar M, McLaughlin PR, et al. Atrial septal defect closure in a patient with "irreversible" pulmonary hypertensive arteriopathy. Int J Cardiol 2006; 110: 104-107.

10 Galiè N, Hoeper MM, Humbert M, et al. Guidelines for the diagnosis and treatment of pulmonary hypertension: the Task Force for the Diagnosis and Treatment of Pulmonary Hypertension of the European Society of Cardiology (ESC) and the European Respiratory Society (ERS), endorsed by the International Society of Heart and Lung Transplantation (ISHLT). Eur Heart J 2009; 30: 2493-2537.

11 Bédard E, Dimopoulos K, Gatzoulis MA. Has there been any progress made on pregnancy outcomes among women with pulmonary arterial hypertension? Eur Heart J 2009; 30: 256-265.

12 Stoica SC, McNeil KD, Perreas K, et al. Heart-lung transplantation for Eisenmenger syndrome: early and long-term results. Ann Thorac Surg 2001; 72: 1887-1891.

13 Waddell TK, Bennett L, Kennedy R, et al. Heart-lung or lung transplantation for Eisenmenger syndrome. J Heart Lung Transplant 2002; 21: 731-737.

14 Berk PD, Goldberg JD, Donovan PB, et al. Therapeutic recommendations in polycythemia vera based on Polycythemia Vera Study Group protocols. Semin Hematol 1986; 23: 132-143.

15 Perloff JK, Marelli AJ, Miner PD. Risk of stroke in adults with cyanotic congenital heart disease. Circulation 1993; 87: 1954-1959.

16 Ammash N, Warnes CA. Cerebrovascular events in adult patients with cyanotic congenital heart disease. J Am Coll Cardiol 1996; 28: 768-772.

17 Diller GP, Dimopoulos K, Broberg CS, et al. Presentation, survival prospects, and predictors of death in Eisenmenger syndrome: a combined retrospective and case-control study. Eur Heart J 2006; 27: 1737-1742.

18 Broberg CS, Bax BE, Okonko DO, et al. Blood viscosity and its relationship to iron deficiency, symptoms, and exercise capacity in adults with cyanotic congenital heart disease. J Am Coll Cardiol 2006; 48: 356-365

19 Galie N, Manes A, Palazzini M, et al. Management of pulmonary arterial hypertension associated with congenital systemic-topulmonary shunts and Eisenmenger's syndrome. Drugs 2008; 68: 1049-1066.

20 Tay EL, Peset A, Papaphylactou M, et al. Replacement therapy for iron deficiency improves exercise capacity and quality of life in patients with cyanotic congenital heart disease and/or the Eisenmenger syndrome. Int J Cardiol 2011; 151: 307-312.

21 Kermeen FD, Franks C, O'Brien K, et al. Endothelin receptor antagonists are an effective long term treatment option in pulmonary arterial hypertension associated with congenital heart disease with or without trisomy 21. Heart Lung Circ 2010; 19: 595-600.

22 Berger RM, Beghetti M, Galiè N, et al. Atrial septal defects versus ventricular septal defects in BREATHE-5, a placebo-controlled study of pulmonary arterial hypertension related to Eisenmenger's syndrome: a subgroup analysis. Int J Cardiol 2010; 144: 373-378.

23 Duffels MG, van der Plas MN, Surie $S$, et al. Bosentan in pulmonary arterial hypertension: a comparison between congenital heart disease and chronic pulmonary embolism. Neth Heart J 2009; 17: 334-338.

24 Diller GP, Dimopoulos K, Kaya MG, et al. Long-term safety, tolerability and efficacy of bosentan in adults with pulmonary arterial hypertension associated with congenital heart disease. Heart 2007; 93: 974-976.

25 Hopkins WE, Ochoa LL, Richardson GW, et al. Comparison of the hemodynamics and survival of adults with severe primary pulmonary hypertension or Eisenmenger syndrome. J Heart Lung Transplant 1996; 15: 100-105.

26 Humbert M, Barst RJ, Robbins IM, et al. Combination of bosentan with epoprostenol in pulmonary arterial hypertension: BREATHE-2. Eur Respir J 2004; 24: 353-359.

27 Hoeper MM, Leuchte H, Halank M, et al. Combining inhaled iloprost with bosentan in patients with idiopathic pulmonary arterial hypertension. Eur Respir J 2006; 28: 691-694.

28 Hoeper MM, Markevych I, Spiekerkoetter E, et al. Goal-oriented treatment and combination therapy for pulmonary arterial hypertension. Eur Respir J 2005; 26: 858-863.

29 Hoeper MM, Faulenbach C, Golpon H, et al. Combination therapy with bosentan and sildenafil in idiopathic pulmonary arterial hypertension. Eur Respir J 2004; 24: 1007-1010.

30 McLaughlin VV, Benza RL, Rubin LJ, et al. Addition of inhaled treprostinil to oral therapy for pulmonary arterial hypertension: a randomized controlled clinical trial. J Am Coll Cardiol 2010; 55: 1915-1922.

31 McLaughlin VV, Oudiz RJ, Frost A, et al. Randomized study of adding inhaled iloprost to existing bosentan in pulmonary arterial hypertension. Am J Respir Crit Care Med 2006; 174: 1257-1263.

32 Simonneau G, Rubin LJ, Galiè N, et al. Addition of sildenafil to long-term intravenous epoprostenol therapy in patients with pulmonary arterial hypertension: a randomized trial. Ann Intern Med 2008; 149: 521-530.

33 D'Alto M, Romeo E, Argiento P, et al. Bosentan-sildenafil association in patients with congenital heart disease-related pulmonary arterial hypertension and Eisenmenger physiology. Int J Cardiol 2010; [Epub ahead of print DOI: 10.1016/j.ijcard. 2010.10.051].

34 Bendayan D, Shitrit D, Kramer MR. Combination therapy with prostacyclin and tadalafil for severe pulmonary arterial hypertension: a pilot study. Respirology 2008; 13: 916-918.

35 Post MC, Janssens S, Van de Werf F, et al. Responsiveness to inhaled nitric oxide is a predictor for mid-term survival in adult patients with congenital heart defects and pulmonary arterial hypertension. Eur Heart J 2004; 25: 1651-1656.

36 D'Alto M, Romeo E, Argiento P, et al. Pulmonary vasoreactivity predicts long-term outcome in patients with Eisenmenger syndrome receiving bosentan therapy. Heart 2010; 96: 1475-1479.

37 Galiè N, Manes A, Negro L, et al. A meta-analysis of randomized controlled trials in pulmonary arterial hypertension. Eur Heart $J$ 2009; 30: 394-403.

38 Dimopoulos K, Inuzuka R, Goletto S, et al. Improved survival among patients with Eisenmenger syndrome receiving advanced therapy for pulmonary arterial hypertension. Circulation 2010; 121: $20-25$. 